\title{
THE GERGONNE POINT GENERALIZED THROUGH CONVEX COORDINATES
}

\author{
J. N. BOYD and P. N. RAYCHOWDHURY
}

(Received 27 May 1997 and in revised form 20 October 1997)

\begin{abstract}
The Gergonne point of a triangle is the point at which the three cevians to the points of tangency between the incircle and the sides of the triangle are concurrent. In this paper, we follow Koneĉný [7] in generalizing the idea of the Gergonne point and find the convex coordinates of the generalized Gergonne point. We relate these convex coordinates to the convex coordinates of several other special points of the triangle. We also give an example of relevant computations.
\end{abstract}

Keywords and phrases. Convex (barycentric) coordinates, cevian, Gergonne point, incircle, Ceva's theorem.

1991 Mathematics Subject Classification. Primary 51N20; Secondary 51N10.

1. Introduction. When cevians of particular significance in the general triangle (medians, angle bisectors, etc.) are concurrent, their common point is often called a special point of the triangle. Such points have always held interest for geometers. In the past, we have discovered the convex coordinates $[7,6]$ of several special points at which cevians from the three vertices are concurrent [3, 2]. We choose the terminology "convex coordinates" rather than the more widely used "barycentric" or "trilinear coordinates" because of their relevance to convex sets [9].

Now, let the circle $C(I)$ be the incircle of $\triangle V_{1} V_{2} V_{3}$ as shown below. The cevians from the vertices to the points of tangency on the opposite sides of the triangle are concurrent at the point $G$ which is known as the Gergonne point of the triangle [5, 8].

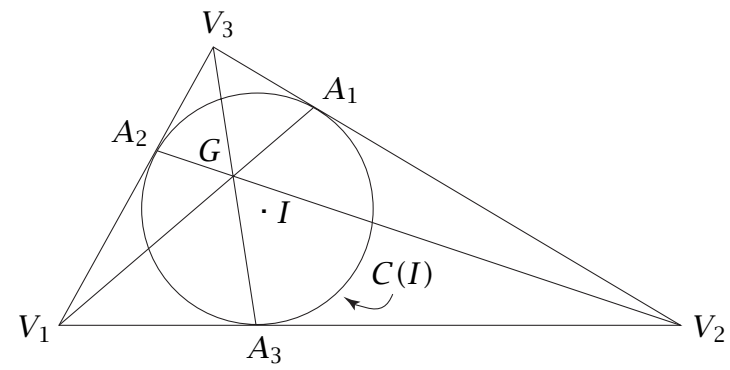

FiguRE 1. Incircle $C(I)$ with Gergonne point $G$ in $\Delta V_{1} V_{2} V_{3}$.

The convex coordinates of a point $P$ in the plane of $\triangle V_{1} V_{2} V_{3}$ and relative to this triangle may be taken as weights which if placed at vertices $V_{1}, V_{2}, V_{3}$, cause $P$ to 
become the balance point for the plane. The plane is taken to be horizontal and otherwise weightless. Also, we require that the sum of the weights have unit value. If $P$ belongs to the closed triangular region, then all the three weights are nonnegative.

We denote the weight placed at vertex $V_{i}$ by $\alpha_{i}$. Then point $P$ has convex coordinates $\left(\alpha_{1}, \alpha_{2}, \alpha_{3}\right)$ with respect to $V_{1}, V_{2}, V_{3}$, in that order, and $\alpha_{1}+\alpha_{2}+\alpha_{3}=1$. For example, the convex coordinates of the vertices $V_{1}, V_{2}, V_{3}$, are $(1,0,0),(0,1,0)$, $(0,0,1)$, respectively, and the convex coordinates of the centroid of $\triangle V_{1} V_{2} V_{3}$ are $\left(\alpha_{1}, \alpha_{2}, \alpha_{3}\right)=(1 / 3,1 / 3,1 / 3)$. All points in the exterior of $\triangle V_{1} V_{2} V_{3}$ must have at least one negative coordinate.

Let us return to the Gergonne point $G$ and consider $\triangle V_{1} V_{2} V_{3}$ with its incircle $C(I)$ as redrawn in Figure 2. The point at which $C(I)$ touches the side opposite to $V_{i}$ is denoted by $A_{i}$. The points of tangency divide the sides into segments of lengths $x_{1}, x_{2}, x_{3}$ as shown in the figure.

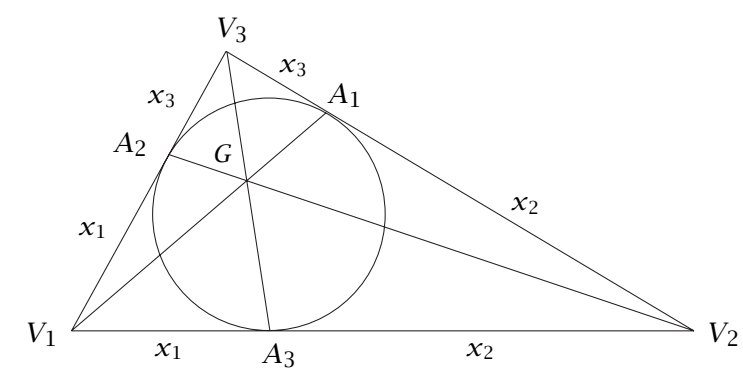

FIGURE 2. $\Delta V_{1} V_{2} V_{3}$, Incircle $C(I)$ and Gergonne point $G$.

It is not a difficult task to show that the convex coordinates of $G$ have the values

$$
\alpha_{1}=\frac{x_{2} x_{3}}{w}, \quad \alpha_{2}=\frac{x_{1} x_{3}}{w}, \quad \alpha_{3}=\frac{x_{1} x_{2}}{w},
$$

where $w=x_{1} x_{2}+x_{2} x_{3}+x_{1} x_{3} \cdot[1]$

The lengths of the sides and measures of the angles of $\triangle V_{1} V_{2} V_{3}$ are more immediately accessible numbers than are $x_{1}, x_{2}$, and $x_{3}$. So, let $\ell_{i}$ denote the length of the side opposite to vertex $V_{i}$ and $\theta_{i}$ denote the measure of the angle at $V_{i}$. Figure 3 depicts the triangle again and establishes the notation for the work to follow.

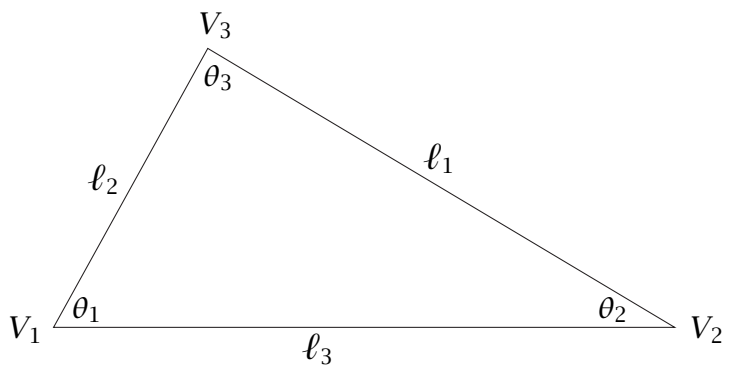

FIGURE 3. $\Delta V_{1} V_{2} V_{3}$ with sides and angles labeled.

The lengths of the sides and the values $x_{1}, x_{2}$, and $x_{3}$ are related by the equations 


$$
\ell_{1}=x_{2}+x_{3}, \quad \ell_{2}=x_{1}+x_{3}, \quad \ell_{3}=x_{1}+x_{2} .
$$

Thus, $x_{1}=\left(\ell_{2}+\ell_{3}-\ell_{1}\right) / 2, x_{2}=\left(\ell_{1}+\ell_{3}-\ell_{2}\right) / 2$, and $x_{3}=\left(\ell_{1}+\ell_{2}-\ell_{3}\right) / 2$. Substitution of these results into the expressions (1) for the convex coordinates of the Gergonne point would not improve their already pleasing appearance.

We have found that convex coordinates provide a straightforward method for investigating special points of triangles.Thus, we read with interest a problem proposed by V. Koneĉný [7] which concerns a generalization of the Gergonne point. In our paper, we find the convex coordinates of Koneĉnýs generalized Gergonne point and, in the process, provide an independent proof that the relevant cevians are concurrent.

2. A generalization of the Gergonne point. Let $I$ be the incenter of $\triangle V_{1} V_{2} V_{3}$ and let $D(I)$ be a circle concentric with incircle $C(I)$ as shown in Figure 4. Suppose that lines are drawn through $I$ perpendicular to the sides of the triangle. These lines intersect the sides of $\triangle V_{1} V_{2} V_{3}$ at $A_{1}, A_{2}, A_{3}$, the points of tangency between the triangle and the incircle and they intersect circle $D(I)$ at points $B_{1}, B_{2}$, and $B_{3}$.

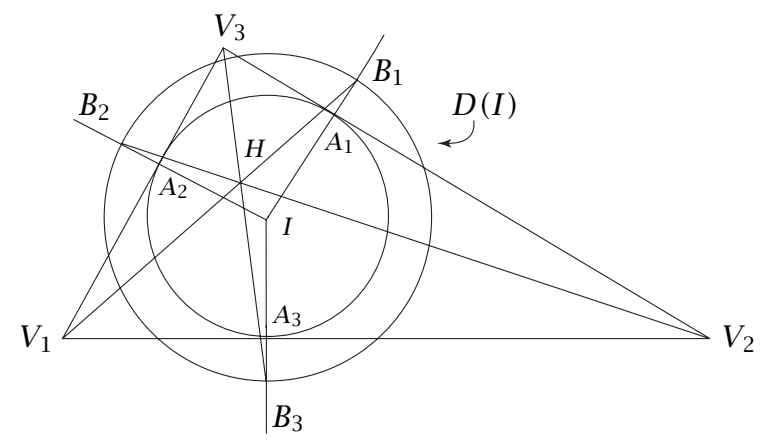

FIGURE 4. $\Delta V_{1} V_{2} V_{3}$ and circle $D(I)$.

Koneĉný's problem is to show that the cevians $\overline{V_{1} B_{1}}, \overline{V_{2} B_{2}}, \overline{V_{3} B_{3}}$ are concurrent. The point $H$ at which the cevians are concurrent is a generalized Gergonne point. We compute the convex coordinates for $H$ and the computational path to our result makes it obvious that the cevians are concurrent.

We begin by noting that there exists $\triangle W_{1} W_{2} W_{3}$ for which $D(I)$ is the incircle and for which $H$ is the Gergonne point. This triangle is similar to $\triangle V_{1} V_{2} V_{3}$; its sides are parallel to the corresponding sides of $\triangle V_{1} V_{2} V_{3}$; and the two triangles are "concentric". If the radius of $C(I)$ is $r>0$, then the radius of $D(I)$ is $r+t$, where $-r<t$. The corresponding sides of the two triangles are a perpendicular distance of $|t|$ units apart and the similarity ratio for lengths in the two triangles is (length in $\triangle W_{1} W_{2} W_{3}$ ): (length in $\left.\triangle V_{1} V_{2} V_{3}\right)=(r+t): r$. The geometry of the two triangles and their incircles is shown in Figure 5.

Points $B_{1}, B_{2}, B_{3}$ divide the sides of $\triangle W_{1} W_{2} W_{3}$ into segments of lengths $y_{1}, y_{2}, y_{3}$ just as $A_{1}, A_{2}, A_{3}$ divide the sides of $\triangle V_{1} V_{2} V_{3}$ into segments of lengths $x_{1}, x_{2}, x_{3}$. An additional crucial observation is that $W_{i}, V_{i}, I$ are collinear for $i=1,2,3$ since rays $\overrightarrow{W_{i} I}$ and $\overrightarrow{V_{i} I}$ bisect the congruent vertex angles at $W_{i}$ and $V_{i}$. 


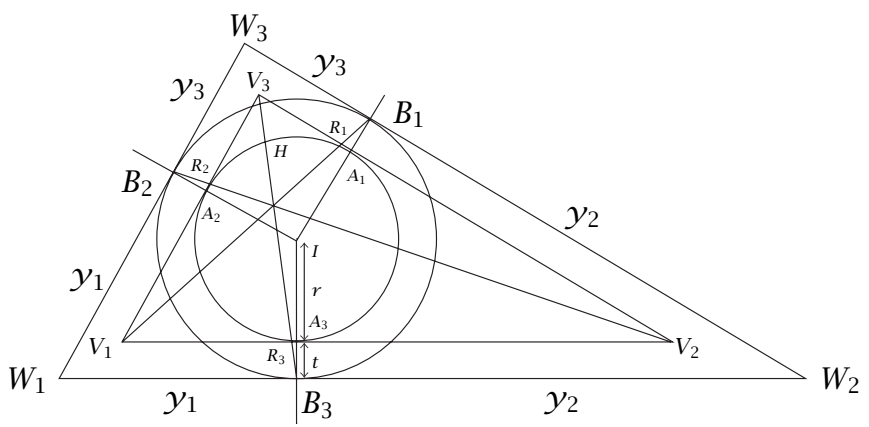

FIGURE 5. The geometry of $\Delta V_{1} V_{2} V_{3}$ and $W_{1} W_{2} W_{3}$.

Let $R_{i}$ be the point at which $\overline{V_{i} B_{i}}$ intersects the side of $\triangle V_{1} V_{2} V_{3}$ opposite to vertex $V_{i}$. We must compute the lengths of $\overline{V_{1} R_{3}}, \overline{V_{2} R_{3}}, \overline{V_{2} R_{1}}, \overline{V_{3} R_{1}}, \overline{V_{3} R_{2}}$, and $\overline{V_{1} R_{2}}$. These segments serve as lever arms when weights $\alpha_{1}, \alpha_{2}, \alpha_{3}$ are placed at $V_{1}, V_{2}, V_{3}$ and the cevians $\overline{V_{1} R_{1}}, \overline{V_{2} R_{2}}, \overline{V_{3} R_{3}}$ are taken to define balance lines. We show the calculations for the length of $\overline{V_{1} R_{3}}$ in some detail and then simply state the other five lengths.

We extend $\overline{V_{1} V_{3}}$ to intersect $\overline{W_{1} W_{2}}$ at point $P$ and we draw angle bisector $\overline{W_{1} I}$ through $V_{1}$ as shown in Figure 6.

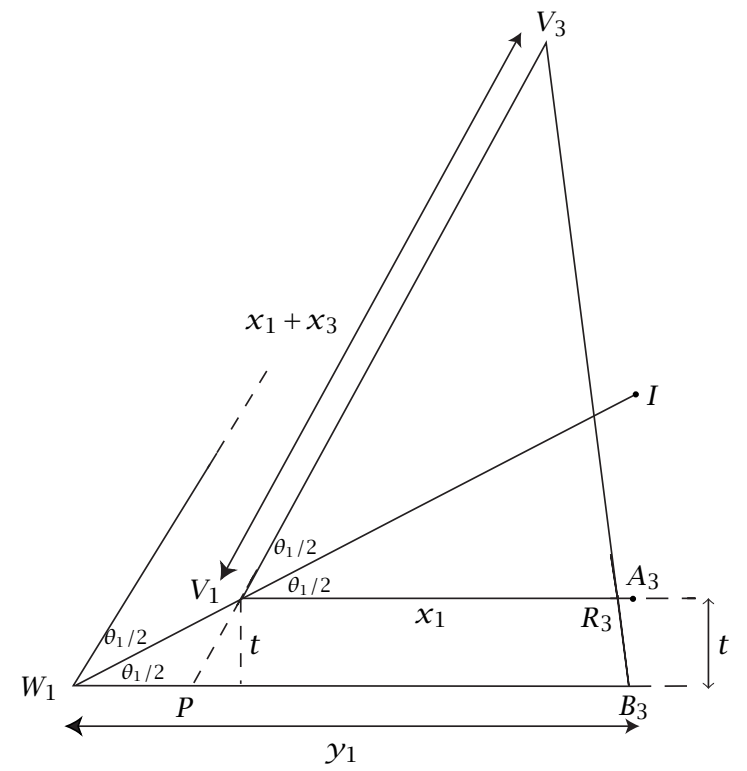

FIGURE 6. The geometry for computing $V_{1} R_{3}$.

Since triangles $W_{1} W_{2} W_{3}$ and $V_{1} V_{2} V_{3}$ are similar with similarity ratio $(r+t): r$, it should be clear that

$$
y_{1}=\left(\frac{r+t}{r}\right) V_{1} A_{3}=\left(\frac{r+t}{r}\right) x_{1}
$$


where the meaning of $x_{i}$ for $i=1,2,3$ is given in Figure 2 . The length of $\overline{P B_{3}}$ is given by

$$
P B_{3}=y_{1}-t\left(\cot \frac{\theta_{1}}{2}-\cot \theta_{1}\right),
$$

and the length of $\overline{P V_{1}}$ by $P V_{1}=t\left(\cot \left(\theta_{1} / 2\right)-\cot \theta_{1}\right)$.

Before proceeding, let us simplify our notation by letting

$$
m_{1}=\left(\cot \frac{\theta_{1}}{2}-\cot \theta_{1}\right) .
$$

Then $P B_{3}=y_{1}-t m_{1}$ and $P V_{1}=t m_{1}$.

Triangles $P V_{3} B_{3}$ and $V_{1} V_{3} R_{3}$ are also similar. Therefore,

$$
V_{1} R_{3}=\frac{\left(x_{1}+x_{3}\right) P B_{3}}{x_{1}+x_{3}+t m_{1}},
$$

where $x_{1}+x_{3}=V_{1} V_{3}=\ell_{2}$.

Appropriate substitutions yield

$$
V_{1} R_{3}=\frac{\left(x_{1}+x_{3}\right)\left[((r+t) / r) x_{1}-t m_{1}\right]}{x_{1}+x_{3}+t m_{1}},
$$

so that all values except $t$ depend only upon the geometry of $\triangle V_{1} V_{2} V_{3}$.

Letting $m_{i}=\cot \left(\theta_{i} / 2\right)-\cot \theta_{i}$ for $i=1,2,3$, we give the results of the other computations for lever arms

$$
\begin{aligned}
& V_{2} R_{3}=\frac{\left(x_{2}+x_{3}\right)\left[((r+t) / r) x_{2}-t m_{2}\right]}{x_{2}+x_{3}+t m_{2}}, \\
& V_{2} R_{1}=\frac{\left(x_{1}+x_{2}\right)\left[((r+t) / r) x_{2}-t m_{2}\right]}{x_{1}+x_{2}+t m_{2}}, \\
& V_{3} R_{1}=\frac{\left(x_{1}+x_{3}\right)\left[((r+t) / r) x_{3}-t m_{3}\right]}{x_{1}+x_{3}+t m_{3}}, \\
& V_{3} R_{2}=\frac{\left(x_{2}+x_{3}\right)\left[((r+t) / r) x_{3}-t m_{3}\right]}{x_{2}+x_{3}+t m_{3}}, \\
& V_{1} R_{2}=\frac{\left(x_{1}+x_{2}\right)\left[((r+t) / r) x_{1}-t m_{1}\right]}{x_{1}+x_{2}+t m_{1}} .
\end{aligned}
$$

At first glance, these expressions seem quite complicated but an examination of the subscripts should reveal their symmetry. The appearance of such symmetry gives confidence in the computations thus far. However, for the next calculations, a few changes in form are helpful. We make the substitutions suggested by equations (1) and (9). The result, denoted by (9), follows from the triangles shown in Figure 7.

We have extended $\overline{V_{3} V_{2}}$ to $B$ on $\overline{W_{1} W_{2}}$ and drawn $\overline{A V_{2}}$ parallel to $\overline{V_{1} V_{3}}$. Thus, $\triangle V_{1} V_{2} V_{3}$ $\sim \triangle A B V_{2}$. The length of $\overline{V_{2} A}$ is $t m_{1}$ and the length of $\overline{V_{2} B}$ is $t m_{2}$. From the similar triangles, we have $t \ell_{2} m_{2}=t \ell_{1} m_{1}$. Taking the smaller triangle at a different vertex of $\triangle V_{1} V_{2} V_{3}$ yields

$$
t \ell_{1} m_{1}=t \ell_{2} m_{2}=t \ell_{3} m_{3}=k
$$




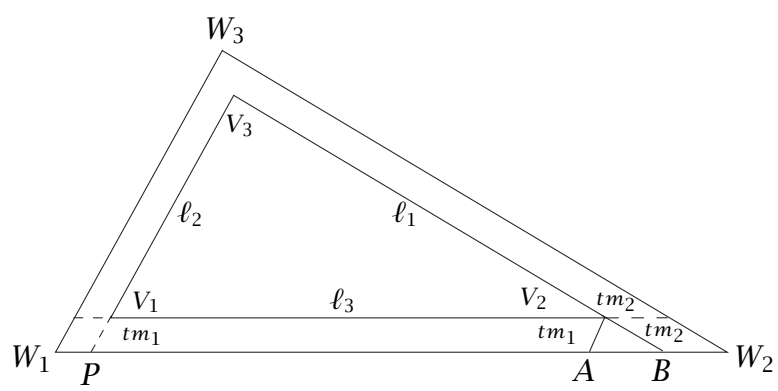

FIGURE 7. Similar triangles $V_{1} V_{2} V_{3}$ and $A B V_{2}$.

Equations (7) and (8) become

$$
\begin{aligned}
& V_{1} R_{3}=\frac{\ell_{2}\left[\ell_{1}((r+t) / r) x_{1}-k\right]}{\ell_{1} \ell_{2}+k}, \\
& V_{2} R_{3}=\frac{\ell_{1}\left[\ell_{2}((r+t) / r) x_{2}-k\right]}{\ell_{1} \ell_{2}+k}, \\
& V_{2} R_{1}=\frac{\ell_{3}\left[\ell_{2}((r+t) / r) x_{2}-k\right]}{\ell_{2} \ell_{3}+k}, \\
& V_{3} R_{1}=\frac{\ell_{2}\left[\ell_{3}((r+t) / r) x_{3}-k\right]}{\ell_{2} \ell_{3}+k}, \\
& V_{3} R_{2}=\frac{\ell_{1}\left[\ell_{3}((r+t) / r) x_{3}-k\right]}{\ell_{1} \ell_{3}+k}, \\
& V_{1} R_{2}=\frac{\ell_{3}\left[\ell_{1}((r+t) / r) x_{1}-k\right]}{\ell_{1} \ell_{3}+k} .
\end{aligned}
$$

It becomes clear at this stage that $\overline{V_{1} R_{1}}, \overline{V_{2} R_{2}}$, and $\overline{V_{3} R_{3}}$ must be concurrent. That conclusion follows from Ceva's theorem since

$$
\frac{V_{1} R_{3}}{V_{2} R_{3}} \cdot \frac{V_{2} R_{1}}{V_{3} R_{1}} \cdot \frac{V_{3} R_{2}}{V_{1} R_{2}}=1 .
$$

To find the convex coordinates of $H$, the generalized Gergonne point at which the cevians are concurrent, we place weights $\alpha_{1}^{\prime}, \alpha_{2}^{\prime}, \alpha_{3}^{\prime}$ at $V_{1}, V_{2}, V_{3}$ respectively and require that the cevians $\overline{V_{3} R_{3}}$ and $\overline{V_{1} R_{1}}$ define balance lines. This means that

$$
\left(V_{1} R_{3}\right) \alpha_{1}^{\prime}=\left(V_{2} R_{3}\right) \alpha_{2}^{\prime}
$$

and

$$
\left(V_{2} R_{1}\right) \alpha_{2}^{\prime}=\left(V_{3} R_{1}\right) \alpha_{3}^{\prime}
$$

The weights are denoted by $\alpha_{i}^{\prime}$ since we will not normalize coordinates until we have convinced ourselves that we have a triple of weights with point $H$ as balance point. Then we write $\alpha_{i}=\alpha_{i}^{\prime} /\left(\alpha_{1}^{\prime}+\alpha_{2}^{\prime}+\alpha_{3}^{\prime}\right)$. 
A bit of algebra suggests that

$$
\begin{aligned}
& \alpha_{1}^{\prime}=\left(V_{2} R_{3}\right)\left(V_{3} R_{1}\right)=\frac{\ell_{1}\left[\ell_{2}((r+t) / r) x_{2}-k\right]}{\ell_{1} \ell_{2}+k} \cdot \frac{\ell_{2}\left[\ell_{3}((r+t) / r) x_{3}-k\right]}{\ell_{2} \ell_{3}+k}, \\
& \alpha_{2}^{\prime}=\left(V_{1} R_{3}\right)\left(V_{3} R_{1}\right)=\frac{\ell_{2}\left[\ell_{1}((r+t) / r) x_{1}-k\right]}{\ell_{1} \ell_{2}+k} \cdot \frac{\ell_{2}\left[\ell_{3}((r+t) / r) x_{3}-k\right]}{\ell_{2} \ell_{3}+k}, \\
& \alpha_{3}^{\prime}=\left(V_{2} R_{1}\right)\left(V_{1} R_{3}\right)=\frac{\ell_{3}\left[\ell_{2}((r+t) / r) x_{2}-k\right]}{\ell_{2} \ell_{3}+k} \cdot \frac{\ell_{2}\left[\ell_{1}((r+t) / r) x_{1}-k\right]}{\ell_{1} \ell_{2}+k} .
\end{aligned}
$$

The reader may satisfy himself that $\alpha_{1}^{\prime}, \alpha_{2}^{\prime}, \alpha_{3}^{\prime}$ do indeed satisfy equations (12) and (13). The two cevians define balance lines and their point of intersection must be the balance point. The third cevian defines a balance line if and only if

$$
\left(V_{3} R_{2}\right) \alpha_{3}^{\prime}=\left(V_{1} R_{2}\right) \alpha_{1}^{\prime}
$$

Substitution of the values for $\alpha_{1}^{\prime}, \alpha_{2}^{\prime}, \alpha_{3}^{\prime}$ into equation (15) yields $\left(V_{3} R_{2}\right)\left(V_{2} R_{1}\right) \times$ $\left(V_{1} R_{3}\right)=\left(V_{1} R_{2}\right)\left(V_{2} R_{3}\right)\left(V_{3} R_{1}\right)$ which holds true if and only if

$$
\frac{V_{1} R_{3}}{V_{2} R_{3}} \cdot \frac{V_{2} R_{1}}{V_{3} R_{1}} \cdot \frac{V_{3} R_{2}}{V_{1} R_{2}}=1 .
$$

This last equation is valid by Ceva's theorem. Thus, the convex coordinates of the generalized Gergonne point $H$ are given by

$$
\alpha_{1}=\frac{\alpha_{1}^{\prime}}{\alpha_{1}^{\prime}+\alpha_{2}^{\prime}+\alpha_{3}^{\prime}}, \quad \alpha_{2}=\frac{\alpha_{2}^{\prime}}{\alpha_{1}^{\prime}+\alpha_{2}^{\prime}+\alpha_{3}^{\prime}}, \quad \alpha_{3}=\frac{\alpha_{3}^{\prime}}{\alpha_{1}^{\prime}+\alpha_{2}^{\prime}+\alpha_{3}^{\prime}},
$$

where the definitions of $\alpha_{1}^{\prime}, \alpha_{2}^{\prime}, \alpha_{3}^{\prime}$ are given by equation (14).

\section{Three checks and an example}

CHeck 1. if $\triangle V_{1} V_{2} V_{3}$ is equilateral, then $x_{1}=x_{2}=x_{3}$ and $\ell_{1}=\ell_{2}=\ell_{3}$ which implies that $\alpha_{1}=\alpha_{2}=\alpha_{3}=1 / 3$ as desired since the symmetry of the triangle forces $H$ to coincide with the centroid.

Check 2. If $t=0, H$ becomes the Gergonne point. Letting $t=0$ means that $k=$ 0 . Then equations (7), (8), and (14) imply that $\alpha_{1}, \alpha_{2}, \alpha_{3}$ have the values given by equations (1).

CHEck 3. If $t \rightarrow-r$. Then $H$ approaches the incenter $I$ of $\Delta V_{1} V_{2} V_{3}$ and the convex coordinates $\left(\alpha_{1}, \alpha_{2}, \alpha_{3}\right)$ approach the convex coordinates of the incenter,

$$
\left(\frac{\ell_{1}}{\ell_{1}+\ell_{2}+\ell_{3}}, \frac{\ell_{2}}{\ell_{1}+\ell_{2}+\ell_{3}}, \frac{\ell_{3}}{\ell_{1}+\ell_{2}+\ell_{3}}\right) \cdot[4]
$$

EXAMPLE. Let $\triangle V_{1} V_{2} V_{3}$ be the 3-4-5 right triangle shown in Figure 8. Observation and a bit of computation yield that $\ell_{1}=5, \ell_{2}=3, \ell_{3}=4, r=$ radius of incircle $C(I)=$ $1, x_{1}=1, x_{2}=3, x_{3}=2, \theta_{1}=90^{\circ}, \cot \theta_{1}=0, \cot \left(\theta_{1} / 2\right)=1$.

Let the radius of $D(I)$ be 2 , which implies that $t=1$. Then $k=t \ell_{1} m_{1}=t \ell_{1}\left(\cot \left(\theta_{1} / 2\right)\right.$ $\left.-\cot \theta_{1}\right)=5$. 


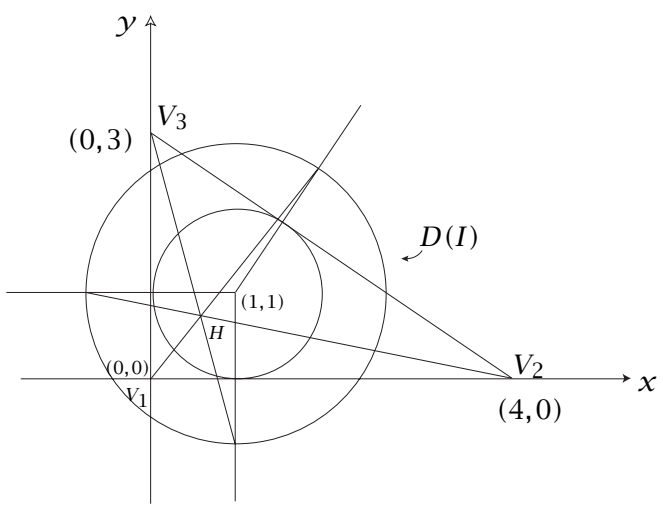

FiguRE 8. The 3-4-5 right triangle located in the Cartesian Plane.

From equations (14) and (15), we obtain the convex coordinates of point $H$ to be $\alpha_{1}=143 / 228, \alpha_{2}=33 / 228, \alpha_{3}=52 / 228$. Then the Cartesian coordinates of $H$ are

$$
\begin{aligned}
(x, y) & =\left(0 \cdot \alpha_{1}+4 \cdot \alpha_{2}+0 \cdot \alpha_{3}, 0 \cdot \alpha_{1}+0 \cdot \alpha_{2}+3 \cdot \alpha_{3}\right) \\
& =\left(4 \cdot \frac{33}{228}, 3 \cdot \frac{52}{228}\right)=\left(\frac{11}{9}, \frac{13}{19}\right) .
\end{aligned}
$$

These Cartesian coordinates can also be found by analytic geometry as a further check upon the accuracy of our computations.

\section{REFERENCES}

[1] J. N. Boyd, Convex coordinates of the Gergonne point, New York State Mathematics Teachers' Journal 35 (1985), 95-98.

[2] J. N. Boyd, J. S. Cooper, and P. N. Raychowdhury, Euclidean geometry from classical mechanics, Internat. J. Math. Ed. Sci. Tech. 20 (1989), 383-390.

[3] J. N. Boyd and P. N. Raychowdhury, Applications of extended convex coordinates, Math. Gaz. 71 (1987), 209-214.

[4] - Random processes with convex coordinates on triangular graphs, Internat. J. Math. Math. Sci. 20 (1997), no. 2, 385-392. MR 97k:60187. Zbl 884.60068.

[5] H. S. M. Coxeter and S. L. Greitzer, Geometry revisited, vol. XIV, Random House: The L. W. Singer Company, New York, 1967. Zbl 166.16402.

[6] M. Hausner, The center of mass and affine geometry, Amer. Math. Monthly 69 (1962), 724737. Zbl 109.38903.

[7] V. Koneĉny, Problem department, Math. Mag. 63 (1990), 130-131.

[8] J. R. Smart, Modern geometry, Brook/Cole Publishing Co., Pacific Grove, California, 1994. Zbl 863.51001.

[9] I. M. Yaglom and V. G. Boltyanskii, Vypuklye figury. [Convex figures.], Gosudarstv. Izdat. Tehn. Teor. Lit., Moscow, Leningrad, 1961 (Russian). MR 14,197d.

BOYd AND RAYCHOWDHURY: DEPARTMENT OF MATHEMATICAL SCIENCES, VIRGINIA COMMONWEALTH UNIVERSITY, RICHMOND, VIRGINIA 23284-2014, USA 


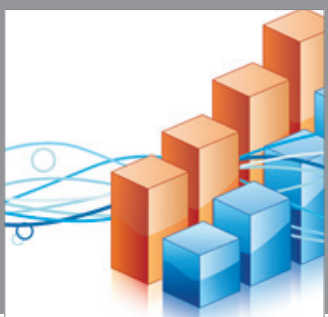

Advances in

Operations Research

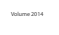

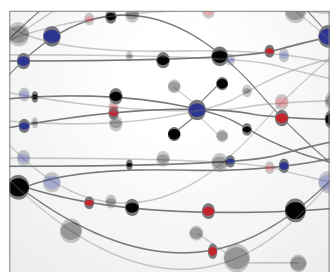

\section{The Scientific} World Journal
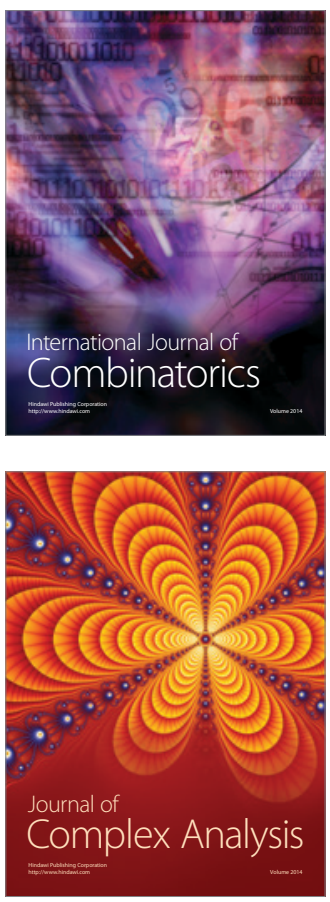

International Journal of

Mathematics and

Mathematical

Sciences
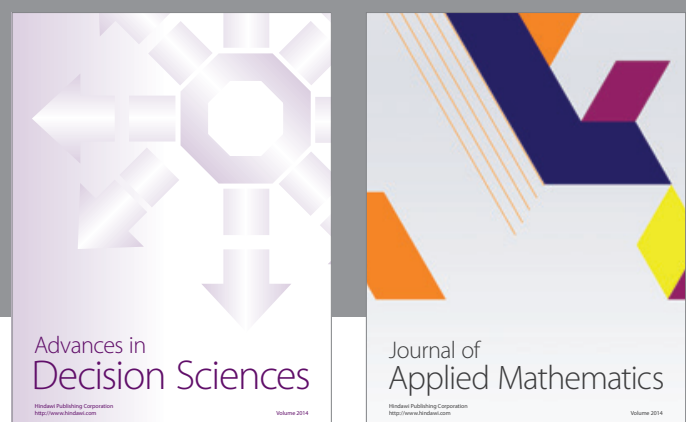

Journal of

Applied Mathematics
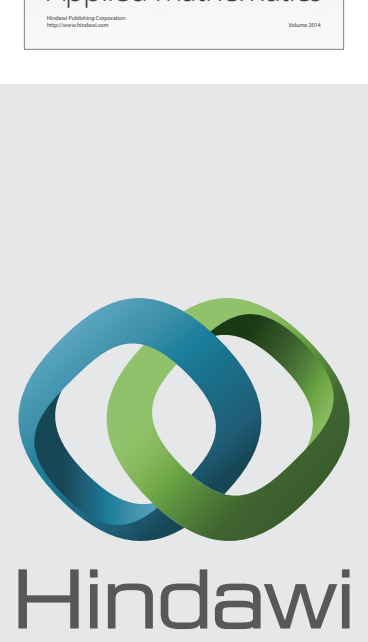

Submit your manuscripts at http://www.hindawi.com
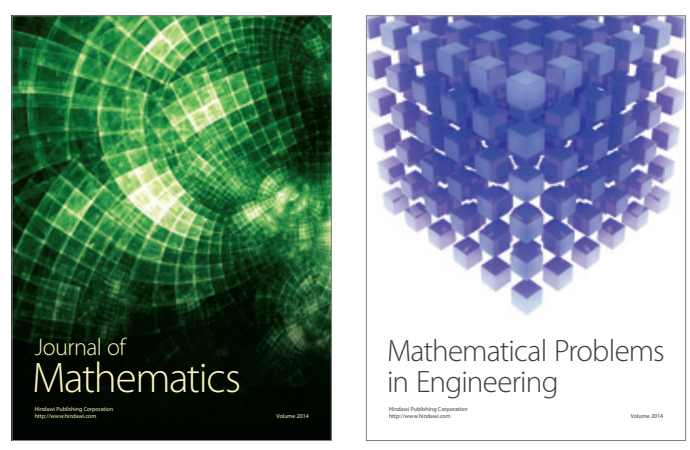

Mathematical Problems in Engineering
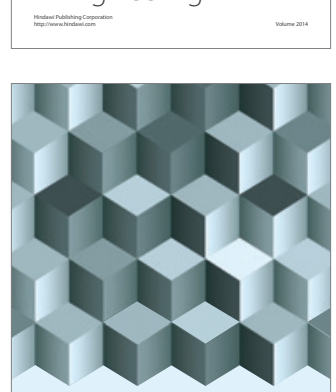

Journal of

Function Spaces
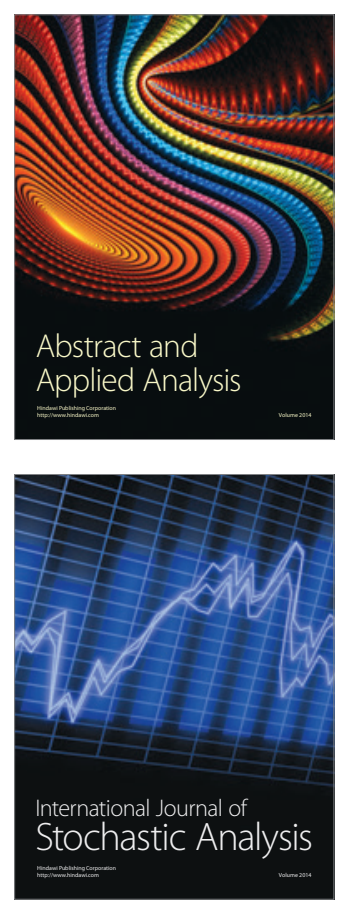

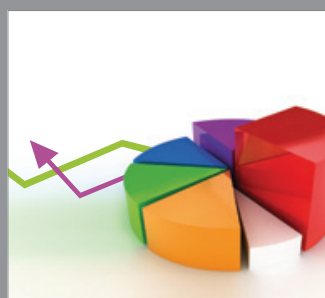

ournal of

Probability and Statistics

Promensencen
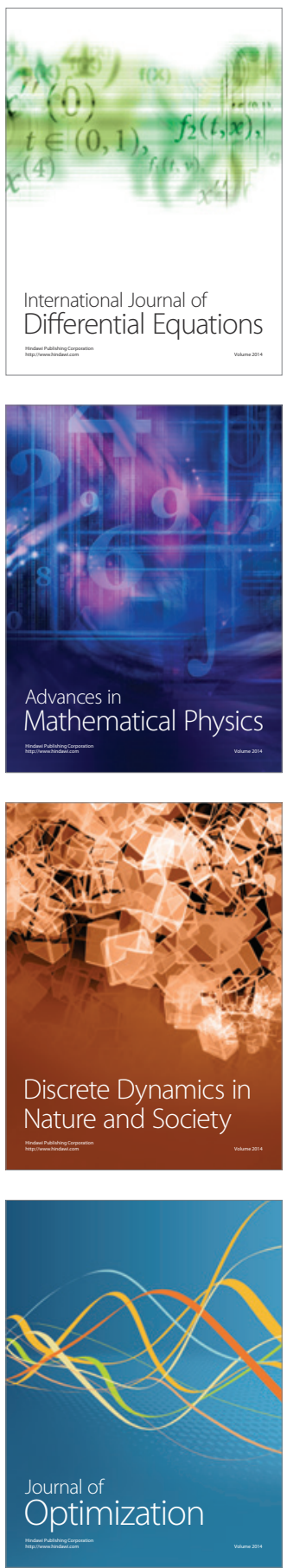